\title{
Baroreflex and Cerebral Autoregulation Are Inversely Correlated
}

\author{
Nathalie Nasr, MD, PhD; Marek Czosnyka, PhD; Anne Pavy-Le Traon, MD, PhD; \\ Marc-Antoine Custaud, MD, PhD; Xiuyun Liu, BSc; \\ Georgios V. Varsos, BSc; Vincent Larrue, MD
}

\begin{abstract}
Background: The relative stability of cerebral blood flow is maintained by the baroreflex and cerebral autoregulation (CA). We assessed the relationship between baroreflex sensitivity (BRS) and CA in patients with atherosclerotic carotid stenosis or occlusion.
\end{abstract}

\begin{abstract}
Methods and Results: Patients referred for assessment of atherosclerotic unilateral $>50 \%$ carotid stenosis or occlusion were included. Ten healthy volunteers served as a reference group. BRS was measured using the sequence method. CA was quantified by the correlation coefficient $(\mathrm{Mx})$ between slow oscillations in mean arterial blood pressure and mean cerebral blood flow velocities from transcranial Doppler. Forty-five patients (M/F: 36/9), with a median age of 68 years (IQR:17) were included. Thirty-four patients had carotid stenosis, and 11 patients had carotid occlusion (asymptomatic: 31 patients; symptomatic: 14 patients). The median degree of carotid steno-occlusive disease was $90 \%(I Q R: 18)$. Both $C A(P=0.02)$ and $B R S(P<0.001)$ were impaired in patients as compared with healthy volunteers. CA and BRS were inversely and strongly correlated with each other in patients ( $\mathrm{rho}=0.58$, $\mathrm{P}<0.001)$ and in healthy volunteers ( $\mathrm{rho}=0.939 ; \mathrm{P}<0.001)$. Increasing $\mathrm{BRS}$ remained strongly associated with impaired CA on multivariate analysis $(\mathrm{P}=0.004)$.
\end{abstract}

Conclusions: There was an inverse correlation between CA and BRS in healthy volunteers and in patients with carotid stenosis or occlusion. This might be due to a relative increase in sympathetic drive associated with weak baroreflex enhancing cerebral vasomotor tone and CA. (Circ J 2014; 78: 2460-2467)

Key Words: Atherosclerosis; Autonomic nervous system; Baroreceptor; Carotid artery; Cerebrovascular circulation

$\mathbf{T}$ he relative stability of cerebral blood flow $(\mathrm{CBF})$ despite fluctuations in blood pressure is maintained by two regulatory mechanisms: the baroreflex and cerebral autoregulation (CA). The baroreflex controls blood pressure in the short term by modulating heart rate, cardiac contractility and vascular tone. ${ }^{1} \mathrm{CA}$ refers to the capacity of cerebral vessels to react through vasodilatation or vasoconstriction to changes in cerebral perfusion pressure that would threaten either to reduce CBF or cause cerebral hyperhemia. Both the baroreflex and CA can be impaired in carotid atherosclerosis. ${ }^{2-4}$ Attenuation of baroreflex sensitivity (BRS) in carotid atherosclerosis is likely to result from the reduced distensibility of carotid bulbs infiltrated by atherosclerosis..$^{5,6}$ Also, it is possible that impaired BRS can accelerate atherosclerosis through inflammation and endothelial cell changes. ${ }^{7,8}$
The mechanism of CA impairment is less clear. CA impairment is usually viewed as a consequence of cerebral vessel dilation secondary to reduced perfusion pressure downstream of the carotid stenosis. ${ }^{9}$

Whether baroreflex and CA are two independent or interdependent mechanisms is not known. Recent data in healthy volunteers showed an inverse correlation between CA and $\mathrm{BRS},{ }^{10}$ while other data did not confirm this inverse correlation. ${ }^{11,12}$ No data are available for a pathological setting such as carotid atherosclerosis.

The purpose of the present study was to test for the correlation between BRS and CA assessed in time domain, using the sequence method for baroreflex, ${ }^{13}$ and the Mx correlation method for CA, ${ }^{14}$ in patients with carotid atherosclerotic stenosis or occlusion and in a group of healthy volunteers.

Received April 16, 2014; revised manuscript received July 10, 2014; accepted July 23, 2014; released online September 4, 2014 Time for primary review: 21 days

Department of Clinical Neurosciences, University of Cambridge, School of Clinical Medicine, Cambridge (N.N.); Academic Neurosurgical Unit, Addenbrooke's Hospital, University of Cambridge, Cambridge (M.C., X.L., G.V.V.), UK; Department of Clinical Neurosciences, University of Toulouse, and INSERM 1048, Toulouse (N.N., A.P.-L.T., V.L.); and Clinical Research Center, University Hospital of Angers, Angers (M.-A.C.), France

Mailing address: Nathalie Nasr, MD, PhD, Unité de Neurologie Vasculaire - Département de Neurologie, CHU de TOULOUSE-Purpan - Hôpital Pierre Paul Riquet - Hall B 2ème étage, TSA 40031, 31059 TOULOUSE cedex 9 - France. E-mail: nathalie.nasr@orange.fr

ISSN-1346-9843 doi:10.1253/circj.CJ-14-0445

All rights are reserved to the Japanese Circulation Society. For permissions, please e-mail: cj@j-circ.or.jp 


\section{Methods}

\section{Subjects}

Carotid Stenosis or Occlusion Patients We retrospectively analyzed data for consecutive patients referred to the Neurosonology Unit of Toulouse University Hospital between January 2007 and July 2012 for assessment of cerebral hemodynamics distal to a carotid stenosis or occlusion.

Patients with unilateral atherosclerotic $\geq 50 \%$ stenosis or occlusion of the cervical internal carotid artery were considered for inclusion.

Exclusion criteria were: $\geq 50 \%$ stenosis or occlusion of contralateral internal carotid artery; $\geq 50 \%$ stenosis or occlusion of the ipsilateral intracranial carotid artery or middle cerebral artery (MCA); insufficient temporal bone acoustic window for transcranial Doppler (TCD) measurements; respiratory failure; history of sleep apnea syndrome; history of brainstem stroke; treatment with $\beta$-blockers, or calcium inhibitors that may increase the QT (diltiazem and verapamil); neurologic disease associated with impairment of the autonomic nervous system.

Carotid stenosis or occlusion was considered symptomatic if the patient had ischemic stroke, transient ischemic attack, or retinal ischemic event ipsilateral to the stenotic/occluded vessel during the previous 2 years.

The study was approved by the institutional review board. Patient consent to participate was not required because the study was retrospective and data had been acquired as part of routine clinical care.

Healthy Volunteers The control group consisted of 10 volunteers taking part in a prospective study that evaluated the cardiovascular and cerebrovascular consequences of microgravity simulation using anti-orthostatic positioning at $-6^{\circ}$ in healthy men. The data we used for this study were collected at baseline, before the volunteers underwent the anti-orthostatic experiment. Before CA was assessed, the ipsilateral cervical carotid artery was screened to rule out atherosclerosis.

The study in healthy volunteers was approved by the institutional review board. Volunteers gave written consent for their participation in the study.

$\mathrm{CA}$ and BRS were assessed using the same methods in healthy volunteers and in patients with carotid stenosis or occlusion.

\section{Assessment of Carotid Stenosis}

Carotid stenosis quantification was made using duplex sonography (Philips, IU22) based on the consensus of the Society of Radiologists in Ultrasound. ${ }^{15}$ The degree of carotid stenosis was confirmed using computed tomography (CT) or magnetic resonance (MR) angiography in all cases. Ruling out intracranial stenosis was based on CT or MR angiography.

Assessment of carotid stenosis using ultrasound was followed by assessment of the circulation in the ophthalmic artery distal to carotid stenosis or occlusion using transcranial color-coded sonography. Reversed circulation in the ipsilateral ophthalmic artery is a marker of severe hemodynamic impact of carotid stenosis or occlusion. ${ }^{16}$

\section{Assessment of CA}

CA was assessed using the Mx method ${ }^{14}$ using TCD in supine patients and healthy volunteers, at rest, during the same period as BRS assessment. The MCA distal to carotid stenosis or occlusion was insonated unilaterally through the temporal bone at a depth of $50-55 \mathrm{~mm}$ with a $2-\mathrm{MHz}$ probe using a DWL Multidop X2 (DWL, Germany). The probe was then fixed using a rigid headframe (Lamrack; DWL). Continuous monitoring of ABP was achieved using a servo-controlled finger plethysmograph (Finapres, Ohmeda, CO, USA for patients with carotid stenosis; and Nexfin, BMI for volunteers). Mean ABP and mean $\mathrm{CBF}$ velocities (CBFV) in the MCA were recorded over 10-20 min. Analog outputs from the pressure monitor and TCD unit (maximal frequency outline) were connected to an analog-to-digital converter and were synchronized. ABP and CBFV signals were collected into a computer and were analyzed using the Mx autoregulatory index.

$\mathrm{Mx}$ is a correlation coefficient derived from the spontaneous slow variations of mean $\mathrm{ABP}$ and mean CBFV. ${ }^{14,17}$ As compared to static and dynamic CA, the Mx method is best described as assessment of semi-static CA because it accounts for slow dynamic components of CA, correlating short- and mid-term variations of $\mathrm{ABP}$ and $\mathrm{CBFV} .{ }^{18}$

Altered CA manifests as an increase in Mx. Mx close to +1 indicates that slow fluctuations in ABP produce synchronized slow changes in CBFV and indicate defective CA. Mx around 0 indicates that variations in $\mathrm{ABP}$ are not associated with variations in $\mathrm{CBFV}$, indicating that $\mathrm{CA}$ is preserved. The magnitude of the increase in Mx reflects the severity of CA impairment. ${ }^{14,17}$ Determination of an absolute cut-off for CA impairment is difficult. The threshold of $\mathrm{Mx}>0.45$ is believed to reasonably characterize CA impairment ${ }^{19}$ and is more specific as compared to the threshold of 0.3 , which has also been used in previous studies. ${ }^{17}$

$\mathrm{Mx}$ has been validated against the Aaslid et al reference method assessing dynamic CA. ${ }^{20,21}$ It also correlated well with the measurement of CA using steady-state methods. ${ }^{17}$

\section{Assessment of BRS}

Resting BRS was measured at rest using the sequence meth$\mathrm{od}^{13}$ with patients lying supine in a quiet room at controlled temperature. Cardiac inter-beat intervals were derived from time in milliseconds between sequential $\mathrm{R}$ spikes on a 3-lead ECG monitor. A peak detection circuit was used to measure each R-R interval to 1-ms accuracy. After each QRS, the subsequent highest and lowest arterial blood pressures were taken as the systolic and diastolic pressures, respectively, from continuous ABP monitoring using servo-controlled finger plethysmograph (Finapres). Finapres ABP was cross-calibrated with an automated Dinamap monitoring system.

The run-time of systolic blood pressure and inter-beat interval monitoring was $10 \mathrm{~min}$. Baroreflex sequences were defined by at least 3 consecutive beats in which the systolic blood pressure and R-R interval of the following beat either increased or decreased by at least $1 \mathrm{mmHg}$ and $5 \mathrm{~ms}$, respectively. Linear regression was applied to each selected sequence and the mean slope was determined as the average of all slopes within a given time period. The mean slope of [inter-beat intervals][systolic blood pressure] variation sequences is considered to be an index of BRS.

\section{Measurement of Cerebrovascular Reactivity}

Assessment of cerebrovascular reactivity was based on the acetazolamide challenge. ABP and CBFV of MCA ipsilateral to carotid stenosis/occlusion were continuously monitored over $10 \mathrm{~min}$ before, and $30 \mathrm{~min}$ after i.v. acetazolamide $13 \mathrm{mg} / \mathrm{kg} .^{22}$

$\mathrm{ABP}$ and $\mathrm{CBFV}$ signals were synchronized, collected into a computer and analyzed using pdl (Notocord Systems, France).

Cerebrovascular reactivity to acetazolamide (CVR-ACZ) was defined as the maximal increase of mean CBFV averaged over 1-min periods up to $30 \mathrm{~min}$ after acetazolamide and was expressed as increase in percentage of baseline mean CBFV. ${ }^{23}$ CVR-ACZ $<25 \%$ was considered as impaired. ${ }^{16,22}$ 


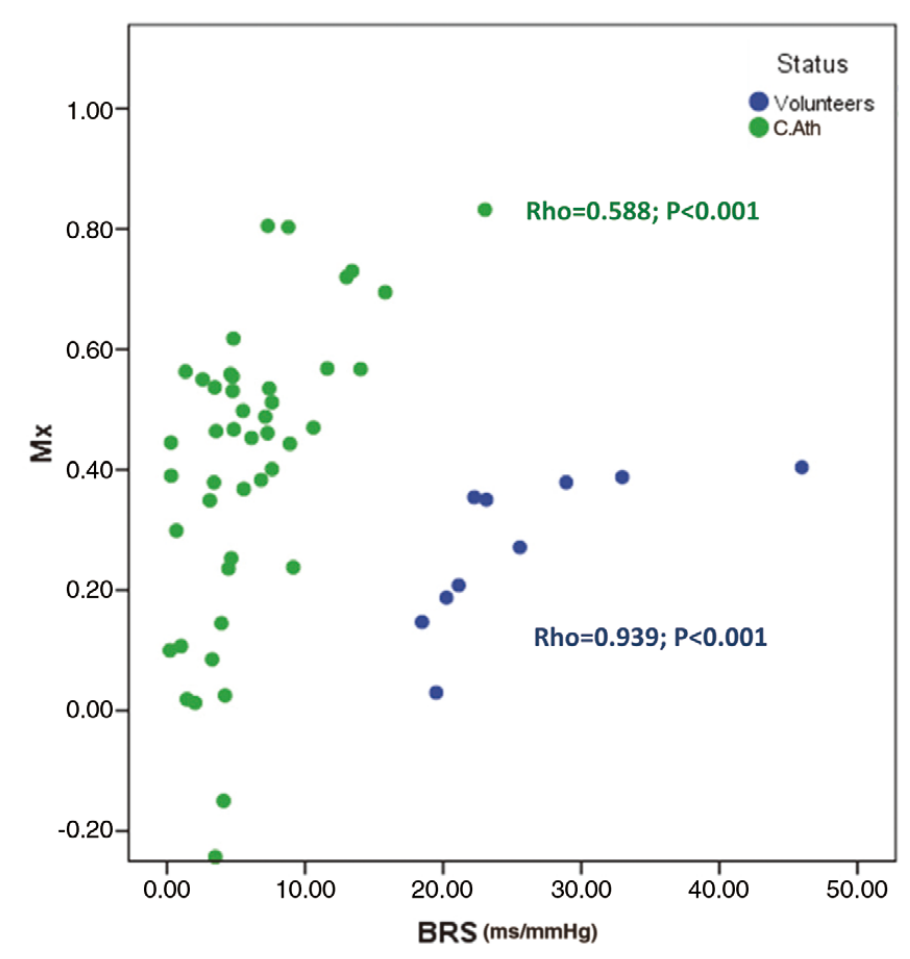

Figure 1. Correlation coefficient between spontaneous $A B P$ and cerebral blood flow velocity variations (Mx) vs. baroreflex sensitivity (BRS; $\mathrm{ms} / \mathrm{mmHg}$ ) assessing, respectively, cerebral autoregulation and efficiency of the baroreflex in healthy volunteers (blue dots; $n=10$ ) and in patients with carotid atherosclerosis (C.Ath; green dots; $n=45$ ). Higher $M x$ indicates worse autoregulation. Higher BRS indicates better baroreflex. Mx was higher $(\mathrm{P}=0.02)$ and $\mathrm{BRS}$ was lower $(P<0.001)$ in patients with carotid stenosis or occlusion as compared to healthy volunteers. $\mathrm{Mx}$ correlated with BRS in healthy volunteers and in patients with carotid atherosclerosis (respectively, rho $=0.939, P<0.001$; rho $=0.588, P<0.001$; Spearman's rank correlation), indicating that worse autoregulation correlated with better BRS.

\section{Statistical Analysis}

Continuous data are given as median (IQR). Comparison of $\mathrm{Mx}$ and BRS in patients with carotid stenosis or occlusion vs. healthy volunteers was done using the Mann-Whitney U-test. Correlation between $\mathrm{CA}(\mathrm{Mx})$ and $\mathrm{BRS}$ as well as correlation between CVR-ACZ and BRS, and correlation between CA and CVR-ACZ were tested using Spearman rank correlation coefficient.

Comparisons between patients with normal CA and those with altered $\mathrm{CA}$, and comparisons between patients with normal CVR-ACZ and those with altered CVR-ACZ were done using Fisher's exact test for discrete variables and the MannWhitney U-test for continuous variables.

In patients with carotid stenosis or occlusion, the association of altered CA (ie, Mx>0.45) with BRS was further assessed on binary logistic regression. Variables associated on univariate analysis with altered $\mathrm{CA}$ with $\mathrm{P}<0.1$ were included. Systematic adjustment was made for age, sex and degree of carotid steno-occlusive disease. Statistical tests were 2-tailed. The level of significance was set at $\mathrm{P}<0.05$.

\section{Results}

\section{Healthy Volunteers}

The control group of healthy volunteers was composed of 10 men aged 20-44 years (median age, 38 years). Median Mx and BRS in this group were, respectively, 0.31 (IQR, 0.204) and $22.69 \mathrm{~ms} / \mathrm{mmHg}$ (IQR, 9.869).

Mx strongly correlated with $\mathrm{BRS}$ (rho=0.939; $\mathrm{P}<0.001$; Figure 1), indicating that enhanced BRS was associated with weaker CA.

Patients With Carotid Stenosis or Occlusion

A total of 45 patients, 36 men and 9 women, with a median age of 68 years (IQR, 17 years) were included. Thirty-four patients had carotid stenosis, and 11 patients had carotid occlusion. The carotid lesion was asymptomatic in 31 patients, and symptomatic in 14 patients. The median degree of carotid steno-occlusive disease was $90 \%$ (IQR, 18\%).

Thirty patients $(68.9 \%)$ had hypertension, 9 patients $(20 \%)$ had diabetes, 29 patients $(64.4 \%)$ had hypercholesterolemia and 14 patients $(31.1 \%)$ were smokers.

Median Mx, CVR-ACZ, and BRS in this group were, respectively, 0.461 (IQR, 0.312), 30.7\% (IQR, 22.5\%) and $4.75 \mathrm{~ms} / \mathrm{mmHg}$ (IQR, $4.265 \mathrm{~ms} / \mathrm{mmHg}$ ).

Patients with carotid stenosis or occlusion had higher $\mathrm{Mx}$ $(\mathrm{P}=0.02)$ and lower BRS $(\mathrm{P}<0.001)$ than the healthy volunteers.

Similarly to healthy volunteers, Mx strongly correlated with $\mathrm{BRS}$ (rho=0.588, $\mathrm{P}<0.001$; Figure 1) in patients with carotid stenosis or occlusion.

Patient characteristics according to CA impairment are summarized in Tables 1,2. The degree of carotid steno-occlusive disease was not correlated with CA impairment (Table 1). There was a non-significant trend toward an association between altered CA and reversed circulation in the ophthalmic artery ipsilateral to carotid stenosis or occlusion $(\mathrm{P}=0.09$; Table 1). CA impairment was strongly associated with higher BRS ( $\mathrm{P}<0.001$; Table 2).

On logistic regression analysis adjusting for age, sex, carotid occlusion vs. stenosis and reversed ophthalmic artery flow, increasing BRS remained strongly associated with altered $\mathrm{CA}$ $(\mathrm{P}=0.004$; Table 3).

CVR-ACZ measured in 40 patients was not correlated with $\mathrm{BRS}$ (rho=-0.182; $\mathrm{P}=0.262$; Figure 2). There was no correlation between Mx and CVR-ACZ (rho=0.004, $\mathrm{P}=0.98$; Figure 3). CVR-ACZ impairment was strongly associated with the degree of carotid steno-occlusive disease $(\mathrm{P}=0.001)$ and with 


\begin{tabular}{lccc|}
\hline Table 1. Subject Characteristics in Relationship With CA Impairment & & \\
& $\begin{array}{c}\text { Normal CA } \\
(\mathbf{n = 2 2})\end{array}$ & $\begin{array}{c}\text { Impaired CA } \\
(\mathbf{n}=\mathbf{2 3})\end{array}$ & P-value $^{\ddagger}$ \\
Age (years) & $61[18]$ & $68[17]$ & 0.2 \\
Male & $17(77.2)$ & $19(82.6)$ & 0.7 \\
Body mass index & $25[4]$ & $25.7[5.6]$ & 0.751 \\
Hypertension & $14(63.6)$ & $17(73.9)$ & 0.6 \\
Diabetes & $6(27.3)$ & $3(13.0)$ & 0.2 \\
Hypercholesterolemia & $15(68.2)$ & $14(60.9)$ & 0.8 \\
Smoking & $6(27.3)$ & $8(34.8)$ & 0.7 \\
ACEl or ARB & $14(63.6)$ & $13(56.5)$ & 0.8 \\
Statins & $18(81.8)$ & $16(69.6)$ & 0.5 \\
Symptomatic stenosis/occlusion & $6(27.3)$ & $8(34.8)$ & 0.7 \\
Degree of carotid disease (\%) & $87.5 \pm 26$ & $90 \pm 15$ & 0.3 \\
Carotid occlusion & $6(27.3)$ & $5(21.7)$ & 0.7 \\
Reversed ophthalmic artery flow & $3(15)$ & $9(40.9)$ & 0.09 \\
\hline
\end{tabular}

Data given as median [IQR] or $\mathrm{n}(\%)$. ${ }^{\dagger} \mathrm{Mx}>0.45$. FFisher's exact test for discrete variables, Mann-Whitney U-test for continuous variables.

ACEI, angiotensin-converting enzyme inhibitor; ARB, angiotensin II receptor blocker; CA, cerebral autoregulation.

\begin{tabular}{|c|c|c|c|}
\hline & $\begin{array}{c}\text { Normal CA } \\
(n=22)\end{array}$ & $\begin{array}{l}\text { Impaired } \mathrm{CA}^{\dagger} \\
(\mathrm{n}=23)\end{array}$ & P-value ${ }^{\ddagger}$ \\
\hline Heart rate (beats/min) & $72.5[15]$ & $68.5[16.8]$ & 0.152 \\
\hline Mean ABP $(\mathrm{mmHg})$ & $101[24]$ & $100[26.8]$ & 0.555 \\
\hline Mean CBFV $(\mathrm{cm} / \mathrm{s})$ & $50.2[26.4]$ & $52.8[20.1]$ & 0.633 \\
\hline $\mathrm{BRS}(\mathrm{ms} / \mathrm{mmHg})$ & $3.73[3.54]$ & 8.25 [6.85] & $<0.001$ \\
\hline
\end{tabular}

Data given as median [IQR]. ${ }^{\dagger} \mathrm{Mx}>0.45$. \#Mann-Whitney U-test.

ABP, arterial blood pressure (measured with Dinamap); BRS, baroreflex sensitivity; CBFV, cerebral blood flow velocities (measured in the middle cerebral artery with transcranial Doppler).

reversed circulation in the ophthalmic artery ipsilateral to carotid stenosis or occlusion $(\mathrm{P}=0.002)$.

\section{Discussion}

BRS and CA were inversely correlated in healthy volunteers and in patients with carotid atherosclerotic stenosis or occlusion: weaker BRS correlated with better CA. This correlation was strongly significant and independent of potential confounders including age.

\section{Inverse Correlation Between BRS and CA}

This finding of inverse correlation between BRS and CA in patients with atherosclerosis is novel because there are no previous reports on such a correlation in a pathological setting.

The inverse correlation found between BRS and CA in healthy volunteers in the present study was found in 1 previous study, by Tzeng et al. ${ }^{10}$ In 19 volunteers, the authors assessed CA using the leg cuff test, and BRS using 3 methods: derived from nitroprusside, derived from phenylephrine and from low-frequency alpha index, and found that dynamic CA was inversely correlated with BRS..$^{10}$

This inverse relation between BRS and CA was not found in 2 other studies in healthy volunteers. ${ }^{11,12}$ In the Ogoh et al study, dynamic CA was assessed in 9 healthy volunteers using the leg cuff test before and after baroreflex suppression achieved using metoprolol and glycopyrrolate. ${ }^{11}$ They found that CA was attenuated after baroreceptor suppression, implying a di-

\begin{tabular}{|lccc|}
\hline \multicolumn{4}{|c|}{ Table 3. Multivariate $^{+}$Predictors of Impaired CA } \\
& OR & $\mathbf{9 5 \%}$ Cl & P-value \\
Age & 1.1 & $1-1.2$ & 0.04 \\
Sex & 1.7 & $0.2-16.1$ & 0.665 \\
Carotid occlusion vs. stenosis & 1.1 & $0.1-10.9$ & 0.906 \\
Reversed ophthalmic artery flow & 4.3 & $0.6-32.3$ & 0.158 \\
BRS & 1.6 & $1.2-2.1$ & 0.004 \\
\hline
\end{tabular}

${ }^{\dagger}$ After adjustment for age, sex, carotid occlusion vs. stenosis and reversed flow in the ipsilateral ophthalmic artery. $¥ \mathrm{Mx}>0.45$. $\mathrm{Cl}$, confidence interval; OR, odds ratio. Other abbreviations as in Tables 1,2.

rect (rather than inverse) relationship between BRS and dynamic CA.

There was a major difference in the experimental protocol between the 2 studies: in the Tzeng et al study, as in the present study, baroreflex was not manipulated and the results were based on correlation between BRS and CA, which do not have absolute indexes and present with individual variations. In contrast, in the Ogoh et al study, acute suppression of BRS was performed in each subject.

The divergent results between the present study and the Tzeng et al study, and the Ogoh et al study could be explained by these large differences in the experimental protocols: acute suppression of baroreflex in the Ogoh et al study ${ }^{11}$ compared 


\section{CVR-ACZ (\%)}

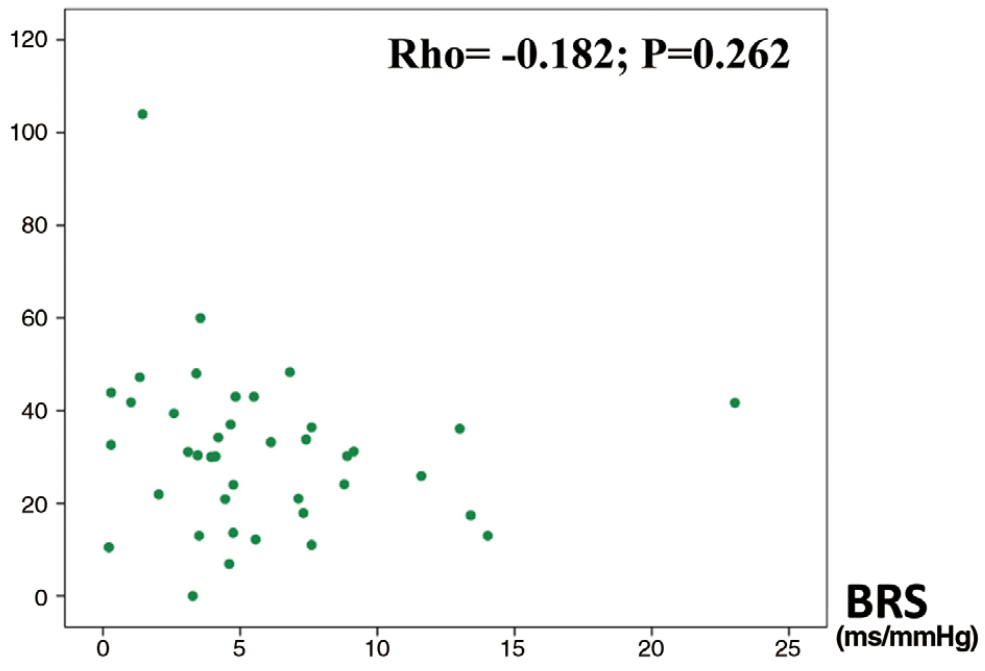

Figure 2. Cerebrovascular reactivity measured as an increase of mean cerebral blood flow velocity in response to acetazolamide injection, expressed as a percentage (CVRACZ) vs. baroreflex sensitivity (BRS; $\mathrm{ms} / \mathrm{mmHg}$ ) in patients with carotid atherosclerosis. Lower CVR-ACZ indicates weaker cerebrovascular reactivity. Lower BRS indicates weaker baroreflex. No correlation was found between CVR-ACZ and BRS (rho= -0.182; $P=0.262$; Spearman's rank correlation coefficient).

\section{CVR-ACZ (\%)}

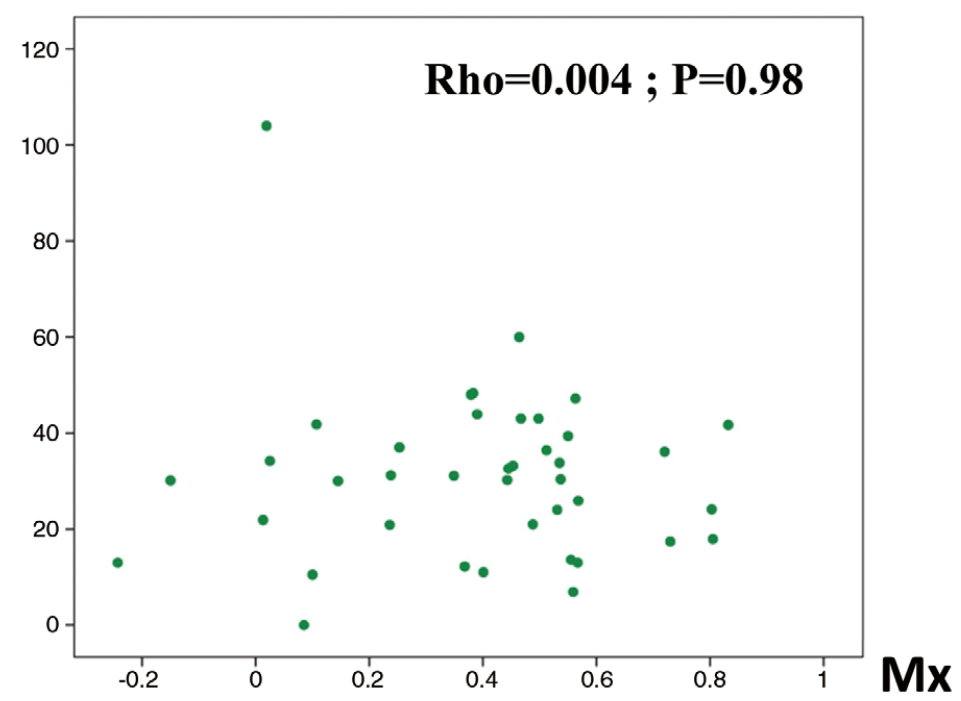

Figure 3. Cerebrovascular reactivity measured as an increase of mean cerebral blood flow velocity (CBFV) in response to acetazolamide injection, expressed as a percentage (CVR-ACZ) vs. the correlation coefficient between spontaneous $\mathrm{ABP}$ and CBFV variations assessing cerebral autoregulation $(C A ; M x)$ in patients with carotid atherosclerosis. Lower CVR-ACZ indicates weaker cerebrovascular reactivity. Higher $\mathrm{Mx}$ indicates weaker CA. No significant correlation was found between CVR-ACZ and CA (rho=0.004, $P=0.98$; Spearman's rank correlation coefficient).

with the intact baroreflex in the Tzeng et al study and in the present study.

In an earlier study, Ogoh et al demonstrated important changes in terms of participation of the vasomotor and cardio-regulatory efference of baroreflex to $\mathrm{ABP}$ regulation when baroreflex is acutely altered, as compared to baroreflex at baseline: participation of the vasomotor efference of baroreflex dropped from $77 \%$ at baseline to $0 \%$ during the first seconds that followed baroreflex impairment. ${ }^{24}$ Therefore, a potential impact of the vasomotor efference of baroreflex on CA could not be demonstrated in the study using acute autonomic blockade. ${ }^{11}$

Aengevaeren et al compared 2 groups: 11 master athletes and 12 healthy sedentary elderly, for BRS and dynamic CA both assessed at rest using the transfer function. Additionally, $\mathrm{CA}$ was also assessed using the sit-stand test. A relationship between BRS and CA was not found. ${ }^{12}$ The Aengevaeren et al study differed from the present one methodologically in that the transfer function used by Aengevaeren et al assessed the rapid properties of $\mathrm{CA}$ rather than its slower dynamic components, which we assessed using Mx. ${ }^{18}$

The present findings of inverse correlation between BRS and $\mathrm{CA}$ in carotid atherosclerosis are novel. Other disease, however, is likely to enhance this correlation. Hypertension is probably the most characteristic example suggesting an in- 
verse correlation between BRS and CA. In hypertension, which is known to be associated with impaired BRS, ${ }^{25}$ most studies have found CA to preserved.26,27 These 2 sets of results in hypertension - low BRS; preserved CA - have been shown in separate studies, but had the correlation between BRS and CA been tested in hypertension, an inverse correlation between BRS and CA could have possibly been found.

\section{CA and CVR-ACZ Impairment Distal to Carotid Stenosis or Occlusion}

The present patients underwent assessment of cerebral hemodynamics distal to an atherosclerotic carotid stenosis or occlusion. Medications known to impair BRS as well as brainstem stroke were exclusion criteria. BRS and CA were assessed simultaneously, at rest. Expectedly, in these patients, BRS and CA were altered as compared to healthy volunteers.

Impaired CA was associated with better BRS while impaired CVR-ACZ was associated with the degree of carotid steno-occlusive disease. CA impairment is a common finding in patients with carotid stenosis or occlusion. CA impairment in these patients has been shown to be weakly associated with the degree of steno-occlusive disease as compared to cerebrovascular reactivity to $\mathrm{CO}_{2}$ or acetazolamide. ${ }^{4}$ In the present study, CA impairment was associated with better BRS and was not associated with the degree of carotid steno-occlusive lesion. Also CA and CVR-ACZ did not correlate. This is in contrast to previous studies that noted moderate correlation between CA impairment, the degree of carotid steno-occlusive lesion and cerebrovascular reactivity to $\mathrm{CO}_{2}{ }^{4,9}$ The fact that we could not reproduce this moderate correlation could be explained by 2 factors: the present sample size was relatively small and, more importantly, we used acetazolamide and not inspired $\mathrm{CO}_{2}$ for assessment of cerebrovascular reactivity. Acetazolamide yields less sensitivity for assessing cerebrovascular reactivity as compared to acetazolamide: an additional increase of cerebral velocities has been observed with $\mathrm{CO}_{2}$ after maximum increase in velocities after acetazolamide injection. ${ }^{28}$

In contrast, we found the expected strong correlation between CVR-ACZ impairment and the degree of carotid stenoocclusive disease. ${ }^{4,16}$ Also, CVR-ACZ was associated with reversed circulation in the ophthalmic artery, which is an indicator of severe hemodynamic impact of the carotid stenosis or occlusion. ${ }^{16}$ In contrast, CA impairment was driven by a better BRS independently of potential confounders, including the degree of carotid steno-occlusive disease.

\section{Mechanisms of Inverse Correlation Between BRS and CA}

One explanation for the inverse correlation between BRS and CA proposed by Tzeng et al is compensation of one homeostatic mechanism by another through central pathways located in the brainstem. ${ }^{10}$ The compensation hypothesis is based on anatomical intersection of the central pathways for BRS and CA. In support of this compensation hypothesis, experimental studies using stimulation or lesion of the tractus solitary nucleus showed resulting modifications in CBF and CA. ${ }^{29,30}$

We propose another potential explanation for this inverse correlation based on the sympathetic drive toward cerebral vasculature. Evidence is building up from physiological studies in humans that the autonomic nervous system strongly influences CA. ${ }^{31-33}$ BRS impairment in patients with carotid atherosclerosis is associated with a deviation of the cardiovascular autonomic balance toward a relative increase of sympathetic activity. ${ }^{3}$ Evidence on the association of baroreflex impairment with an increase of the efferent sympathetic activity to the cerebral vasculature is available from data in rabbits showing an increase in sympathetic activity recorded in the sympathetic cervical trunk after baroreflex deactivation. ${ }^{34}$ Sympathetic activity has been shown to increase the cerebrovascular vasomotor tone, ${ }^{35,36}$ which is the main parameter influencing CA. ${ }^{37}$

Vagal activity has also been shown to affect cerebral circulation and $\mathrm{CA}^{33,38,39}$ and is more associated with BRS than sympathetic activity. ${ }^{40}$ Decrease in vagal activity associated with low BRS in carotid atherosclerosis could thus result in theory in lower CA. Recent data from Hamner and Tan, however, showed sympathetic activity to be the second largest contributor to CA, after the myogenic response. ${ }^{33}$ The cholinergic system contributed in a relatively small but significant fashion. ${ }^{33}$

We thus hypothesize that the inverse correlation between BRS and CA could be explained by enhancement of CA driven by a relative increase in sympathetic activity associated with impaired BRS. This relative increase in sympathetic activity potentially overrides the weaker contribution of vagal retrieval, resulting in increased vasomotor tone and enhanced CA.

\section{Clinical Implications}

The inverse correlation between BRS and CA may have clinical and therapeutic implications. Patients with carotid stenosis or occlusion will need a comprehensive cerebrovascular and cardiovascular assessment taking into account, in addition to cerebral hemodynamics, the baroreflex function, given that BRS is associated with stroke prognosis. ${ }^{41,42}$ Further studies should be done to assess the prognostic value of the interplay between the autonomic nervous system and CA for cerebral ischemic disease, similarly to studies on ischemic heart disease. $^{43}$

Also, BRS impairment could be a therapeutic target for preventing the development of atherosclerosis, given that it could be both cause and result of the development of the atherosclerosis process. BRS impairment may not be the mere consequence of the blunting of the afferent signals from baroreceptors due to building atheroma. Experimental data are available showing that baroreflex impairment can cause atheroma through inflammation and endothelial cell changes. ${ }^{7,8}$ Improvement of baroreflex function after low-dose ketanserin has prevented the development of atherosclerosis in rats and rabbits. This effect was independent of ABP lowering. ${ }^{44}$ Also, in experimental studies on hypertensive rats, the restoration of BRS yielded protection against end-organ damage and against cognitive impairment. ${ }^{45,46}$ Thus, improvement of baroreflex function could be viewed as a potential therapeutic target not only in stroke ${ }^{47}$ but also in situations involving risk for cerebrovascular disease.

In humans, pharmacological and non-pharmacological interventions have been shown to improve baroreflex function. Pharmacological interventions include $\beta$-blockers, low-dose ketanserin, clonidine, moxonidine and mecobalamin..$^{47}$ Nonpharmacological interventions include physical training ${ }^{48}$ and direct electric stimulation of carotid baroreceptors with an implantable device. ${ }^{49}$

\section{Study Limitations}

The control group was composed of a small number of healthy volunteers $(n=10)$ who were not matched for age or sex with patients with carotid atherosclerotic stenosis or occlusion. The control group was younger than the patient group. Expectedly, in the control group, BRS was higher and Mx was lower as 
compared to the patients with carotid stenosis or occlusion. The aim of the study, however, did not pertain to this comparison. The aim was to assess the relationship between baroreflex and CA within the patient group and within the healthy volunteer group as a reference, using the same methods for baroreflex and CA assessment in both groups.

The use of TCD for CBF monitoring requires the diameter of the MCA to remain constant during the tests so variations of $\mathrm{CBFV}$ can be interpreted as variations of $\mathrm{CBF}$. This potential limitation is inherent to all CA studies using TCD. A previous study using $\mathrm{ABP}$ variations during neurosurgical procedures showed that the diameter of the trunk of the MCA does not significantly change during ABP variations. ${ }^{50}$ The MCA diameter has also been found to be stable during ABP variation in an MRI study. ${ }^{51}$

$\mathrm{CO}_{2}$ was not monitored during the tests. $\mathrm{CO}_{2}$ is a potent cerebral vasodilator and its variations might have influenced the results of CA assessment. The Mx method, however, is based on averaging variations of ABP and CBFV over serial periods of time, ${ }^{14}$ which probably reduces the impact of episodic $\mathrm{CO}_{2}$ variations - due to sighs for instance - on CBFV. Also, we excluded patients with clinical conditions associated with hypercapnia.

\section{Conclusions}

We found an inverse correlation between BRS and CA in healthy controls and in patients with carotid stenosis or occlusion. This inverse correlation might be due to baroreflex modulation of the autonomic drive to the cerebral vasculature.

\section{Acknowledgments}

We thank the French Institute for Space Medicine and Physiology (MEDES) for their logistic support in performing the study in healthy volunteers. We also thank Dr Joseph Donnelly for assistance with the manuscript. The National French Center for Space Studies (CNES) and the European Space Agency (ESA) funded the study performed in healthy volunteers.

\section{Disclosures}

This work was supported by a grant from la Direction de la Recherche Clinique et de l'Innovation (DRCI) at Toulouse University Hospital in France.

\section{References}

1. Benarroch EE. The arterial baroreflex: Functional organization and involvement in neurologic disease. Neurology 2008; 71: 1733-1738.

2. Chao AC, Chern CM, Kuo TB, Chou CH, Chuang YM, Wong WJ, et al. Noninvasive assessment of spontaneous baroreflex sensitivity and heart rate variability in patients with carotid atherosclerosis. Cerebrovasc Dis 2003; 16: 151-157.

3. Nasr N, Pavy-Le Traon A, Larrue V. Baroreflex sensitivity is impaired in bilateral carotid atherosclerosis. Stroke 2005; 36: 1891-1895.

4. Reinhard M, Roth M, Muller T, Czosnyka M, Timmer J, Hetzel A. Cerebral autoregulation in carotid artery occlusive disease assessed from spontaneous blood pressure fluctuations by the correlation coefficient index. Stroke 2003; 34: 2138-2144.

5. Chapleau MW, Cunningham JT, Sullivan MJ, Wachtel RE, Abboud FM. Structural versus functional modulation of the arterial baroreflex. Hypertension 1995; 26: 341-347.

6. Tomiyama H, Matsumoto C, Kimura K, Odaira M, Shiina K, Yamashina A. Pathophysiological contribution of vascular function to baroreflex regulation in hypertension. Circ J 2014; 78: $1414-$ 1419.

7. Cai GJ, Miao CY, Xie HH, Lu LH, Su DF. Arterial baroreflex dysfunction promotes atherosclerosis in rats. Atherosclerosis 2005; 183: $41-47$.

8. Shen FM, Zhang SH, Xie HH, Jing Q, Wang DS, Su DF. Early structural changes of aortic wall in sinoaortic-denervated rats. Clin Exp Pharmacol Physiol 2006; 33: 358-363.
9. White R, Markus H. Impaired dynamic cerebral autoregulation in carotid artery stenosis. Stroke 1997; 28: 1340-1344.

10. Tzeng YC, Lucas SJ, Atkinson G, Willie CK, Ainslie PN. Fundamental relationships between arterial baroreflex sensitivity and dynamic cerebral autoregulation in humans. J Appl Physiol 2010; 108: $1162-1168$.

11. Ogoh S, Tzeng YC, Lucas SJ, Galvin SD, Ainslie PN. Influence of baroreflex-mediated tachycardia on the regulation of dynamic cerebral perfusion during acute hypotension in humans. J Physiol 2010; 588: $365-371$.

12. Aengevaeren VL, Claassen JA, Levine BD, Zhang R. Cardiac baroreflex function and dynamic cerebral autoregulation in elderly Masters athletes. J Appl Physiol 2013; 114: 195-202.

13. Parati G, Di Rienzo M, Bertinieri G, Pomidossi G, Casadei R, Groppelli A, et al. Evaluation of the baroreceptor-heart rate reflex by 24-hour intra-arterial blood pressure monitoring in humans. Hypertension 1988; 12: 214-222.

14. Czosnyka M, Smielewski P, Kirkpatrick P, Menon DK, Pickard JD. Monitoring of cerebral autoregulation in head-injured patients. Stroke 1996; 27: 1829-1834.

15. Grant EG, Benson CB, Moneta GL, Alexandrov AV, Baker JD, Bluth EI, et al. Carotid artery stenosis: Gray-scale and Doppler US diagnosis: Society of Radiologists in Ultrasound Consensus Conference. Radiology 2003; 229: 340-346.

16. Kleiser B, Widder B. Course of carotid artery occlusions with impaired cerebrovascular reactivity. Stroke 1992; 23: 171-174.

17. Lang EW, Mehdorn HM, Dorsch NW, Czosnyka M. Continuous monitoring of cerebrovascular autoregulation: A validation study. $J$ Neurol Neurosurg Psychiatry 2002; 72: 583-586.

18. Aaslid R. Cerebral autoregulation and vasomotor reactivity. Front Neurol Neurosci 2006; 21: 216-228.

19. Brady K, Joshi B, Zweifel C, Smielewski P, Czosnyka M, Easley $\mathrm{RB}$, et al. Real-time continuous monitoring of cerebral blood flow autoregulation using near-infrared spectroscopy in patients undergoing cardiopulmonary bypass. Stroke 2010; 41: 1951-1956.

20. Aaslid R, Lindegaard KF, Sorteberg W, Nornes H. Cerebral autoregulation dynamics in humans. Stroke 1989; 20: 45-52.

21. Czosnyka M, Smielewski P, Lavinio A, Pickard JD, Panerai R. An assessment of dynamic autoregulation from spontaneous fluctuations of cerebral blood flow velocity: A comparison of two models, index of autoregulation and mean flow index. Anesth Analg 2008; 106: $234-239$.

22. Grossmann WM, Koeberle B. The dose-response relationship of acetazolamide on the cerebral blood flow in normal subjects. Cerebrovasc Dis 2000; 10: 65-69.

23. Dahl A, Russell D, Rootwelt K, Nyberg-Hansen R, Kerty E. Cerebral vasoreactivity assessed with transcranial Doppler and regional cerebral blood flow measurements: Dose, serum concentration and time course of the response to acetazolamide. Stroke 1995; 26: 2302-2306.

24. Ogoh S, Fadel PJ, Nissen P, Jans O, Selmer C, Secher NH, et al. Baroreflex-mediated changes in cardiac output and vascular conductance in response to alterations in carotid sinus pressure during exercise in humans. J Physiol 2003; 550: 317-324.

25. Head GA. Baroreflexes and cardiovascular regulation in hypertension. J Cardiovasc Pharmacol 1995; 26(Suppl 2): S7-S16.

26. Serrador JM, Sorond FA, Vyas M, Gagnon M, Iloputaife ID, Lipsitz LA. Cerebral pressure-flow relations in hypertensive elderly humans: Transfer gain in different frequency domains. J Appl Physiol 2005; 98: $151-159$.

27. Lipsitz LA, Mukai S, Hamner J, Gagnon M, Babikian V. Dynamic regulation of middle cerebral artery blood flow velocity in aging and hypertension. Stroke 2000; 31: 1897-1903.

28. Kleiser B, Scholl D, Widder B. Assessment of cerebrovascular reactivity by Doppler $\mathrm{CO}_{2}$ and Diamox testing: Which is the appropriate method? Cerebrovasc Dis 1994; 4: 134-138.

29. Nakai M, Tamaki K, Maeda M. Sympathetic and metabolic mechanisms of the cerebrovasomotor function of the caudal ventrolateral medulla in rats. Neuroscience 1992; 50: 655-662.

30. Ishitsuka T, Iadecola C, Underwood MD, Reis DJ. Lesions of nucleus tractus solitarii globally impair cerebrovascular autoregulation. Am J Physiol 1986; 251: H269-H281.

31. Zhang R, Zuckerman J, Iwasaki K, Wilson T, Crandall C, Levine B. Autonomic neural control of dynamic cerebral autoregulation in humans. Circulation 2002; 106: 1814-1820.

32. Ogoh S, Brothers RM, Eubank WL, Raven PB. Autonomic neural control of the cerebral vasculature: Acute hypotension. Stroke 2008; 39: $1979-1987$.

33. Hamner JW, Tan CO. Relative contributions of sympathetic, cholinergic, and myogenic mechanisms to cerebral autoregulation. Stroke 2014; 45: $1771-1777$. 
34. Tafil-Klawe M, Klawe J, Majcherczyk S, Trzebski A. Sympathoinhibitory baroreflex in conscious rabbits: Simultaneous recordings of sympathetic and aortic nerve activity. J Auton Nerv Syst 1989; 28: $227-232$.

35. Van Lieshout JJ, Pott F, Madsen PL, van Goudoever J, Secher NH. Muscle tensing during standing: Effects on cerebral tissue oxygenation and cerebral artery blood velocity. Stroke 2001; 32: 1546-1551.

36. Hamel E. Perivascular nerves and the regulation of cerebrovascular tone. J Appl Physiol 2006; 100: 1059-1064.

37. Paulson OB, Strandgaard S, Edvinsson L. Cerebral autoregulation. Cerebrovasc Brain Metab Rev 1990; 2: 161-192.

38. Seifert T, Fisher JP, Young CN, Hartwich D, Ogoh S, Raven PB, et al. Glycopyrrolate abolishes the exercise-induced increase in cerebral perfusion in humans. Exp Physiol 2010; 95: 1016-1025.

39. Hamner JW, Tan CO, Tzeng YC, Taylor JA. Cholinergic control of the cerebral vasculature in humans. J Physiol 2012; 590: 6343-6352.

40. Ogoh S, Fisher JP, Dawson EA, White MJ, Secher NH, Raven PB. Autonomic nervous system influence on arterial baroreflex control of heart rate during exercise in humans. J Physiol 2005; 566: $599-611$.

41. Robinson TG, Dawson SL, Eames PJ, Panerai RB, Potter JF. Cardiac baroreceptor sensitivity predicts long-term outcome after acute ischemic stroke. Stroke 2003; 34: 705-712.

42. Sykora M, Diedler J, Rupp A, Turcani P, Rocco A, Steiner T. Impaired baroreflex sensitivity predicts outcome of acute intracerebral hemorrhage. Crit Care Med 2008; 36: 3074-3079.

43. Watanabe S, Amiya E, Watanabe M, Takata M, Ozeki A, Watanabe A, et al. Simultaneous heart rate variability monitoring enhances the predictive value of flow-mediated dilation in ischemic heart disease. Circ J 2013; 77: 1018-1025.

44. Yu YS, Xie HH, Li L, Song SW, Han P, Cai GJ, et al. Effects of low-dose ketanserin on atherosclerosis in rats and rabbits. Can J Physiol Pharmacol 2010; 88: 1054-1060.

45. Xie HH, Shen FM, Zhang XF, Jiang YY, Su DF. Blood pressure variability, baroreflex sensitivity and organ damage in spontaneously hypertensive rats treated with various antihypertensive drugs. Eur J Pharmacol 2006; 543: 77-82.

46. Kishi T, Sunagawa K. Combination therapy of atorvastatin and amlodipine inhibits sympathetic nervous system activation and improves cognitive function in hypertensive rats. Circ J 2012; 76: 1934-1941.

47. Sykora M, Diedler J, Turcani P, Hacke W, Steiner T. Baroreflex: A new therapeutic target in human stroke? Stroke 2009; 40: e678e682, doi:10.1161/STROKEAHA.109.565838.

48. Rowaiye OO, Jankowska EA, Ponikowska B. Baroreceptor sensitivity and diabetes mellitus. Cardiol J 2013; 20: 453-463.

49. Uppuluri SC, Storozynsky E, Bisognano JD. Baroreflex device therapy in the treatment of hypertension. Curr Hypertens Rep 2009; 11: $69-75$.

50. Giller CA, Bowman G, Dyer H, Mootz L, Krippner W. Cerebral arterial diameters during changes in blood pressure and carbon dioxide during craniotomy. Neurosurgery 1993; 32: 737-741.

51. Serrador JM, Sorond FA, Vyas M, Gagnon M, Iloputaife ID, Lipsitz LA. Cerebral pressure-flow relations in hypertensive elderly humans: Transfer gain in different frequency domains. J Appl Physiol 2005; 98: $151-159$. 